The findings shift the paradigm of exclusive PLP responsiveness of patients with PNPO deficiency and contradict the strategy of using PLP instead of pyridoxine as the first-line vitamin to test for all inborn errors with vitamin B6-responsive seizures. Testing for PNPO mutations is important in pyridoxine-responsive patients with normal biomarkers for antiquitin deficiency or other B6-dependent neonatal epilepsies. A sequential trial with pyridoxine and PLP should be performed routinely in neonates with AED-resistant seizures, irrespective of a history of birth asphyxia. The challenge of recognizing a delayed pyridoxine effect and lack of specific biomarkers caries a risk of misdiagnosis. (Plecko B, Paul K, Mills P, et al. Pyridoxine responsiveness in novel mutations of the PNPO gene. Neurology 2014 Apr 22;82(16):1425-33).

COMMENTARY. In an editorial [1], the clinical characteristics of pyridoxine dependent epilepsy (PDE) and PLP-dependent cases are differentiated by their presentation with full-term and premature birth, respectively. The EEG of PDE has a classic burst-suppression pattern, whereas the PNPO deficiency is associated with a nonspecific and less paroxysmal pattern. Cases of partial pyridoxine responsiveness have PNPO mutations.

Congenital brain malformations and pyridoxine dependent epilepsy. Several cases of brain malformation are reported in association with pyridoxine dependent epilepsy (PDE), and a current case-report concerns an infant with seizures at 7 days, initially responsive to phenobarbital, later diagnosed with PDE caused by ALDH7A1 genetic defect. Brain sonography on day 1 and MRI on day 5 confirmed bilateral asymmetric ventriculomegaly caused by bilateral subependymal cysts. At age 7 months he was seizure free on pyridoxine $200 \mathrm{mg} /$ day $(29 \mathrm{mg} / \mathrm{kg}$ per day), neurological examination was unremarkable, EEG was normal, and ventriculomegaly was resolved. Despite the structural brain malformation, PDE should always be considered in the differential diagnosis of neonatal seizures refractory to treatment with AEDs [2].

\title{
References.
}

1. Pearl PL, Gospe SM Jr. Neurology. 2014 Apr 22;82(16):1392-4.

2. Jain-Ghai S, et al. Pediatrics. 2014 Apr;133(4):e1092-6.

\section{ETHOSUXIMIDE VS VALPROATE LONG-TERM REMISSION OF ABSENCE EPILEPSY}

Investigators from the Epilepsy Center, Lurie Children's Hospital of Chicago, and Yale School of Medicine, New Haven, CT, examined the possible association between long-term seizure outcome of childhood absence epilepsy (CAE) and the initial treatment with ethosuximide (ESM) or valproic acid (VPA). Newly diagnosed patients throughout the State of Connecticut from 1993 to 1997 were identified through the offices of 16 of 17 child neurologists and data were also reviewed by three pediatric epileptologists. Initial success rates were $59 \%$ of 41 treated with ESM and $56 \%$ of 18 who received VPA. Early remission and drug resistance were similar in each group. Complete remission (seizure free for 5 years and 5 years off medication) occurred in $31(76 \%)$ treated with ESM and $7(39 \%)$ who received VPA ( $p=0.007)$. ESM was associated with a higher rate of complete remission than VPA. In 53 children followed $>10$ years, remission was $76 \%$ 
in the ESM group versus $44 \%$ receiving VPA ( $\mathrm{p}=0.06)$. Atypical EEG features including polyspike-wave and focal findings, occurring in 17\% ESM- and 61\% VPA-treated, did not independently predict outcome $(\mathrm{p}=0.15)$. However, children with atypical EEG features versus those without were less likely to remit completely ( $50 \%$ vs $71 \%, \mathrm{p}=0.03)$. Outcome was associated with initial treatment in patients with or without atypical EEG features; a higher proportion of patients achieved complete remission if first treated with ESM versus VPA. Five-year and 10-year remission, regardless of continued treatment, occurred more often in children initially treated with ESM versus VPA. These clinical findings are congruent with laboratory studies in genetic absence rat models and are supportive of potential disease-modifying effects of ESM in CAE. (Berg AT, Levy SR, Testa FM, Blumenfeld H. Long-term seizure remission in childhood absence epilepsy: Might initial treatment matter? Epilepsia 2014 Apr;55(4):551-7).

COMMENTARY. A disease-modifying or "curative" effect of ethosuximide in children with CAE is an interesting and novel concept in the drug treatment of epilepsy. For complete remission, the characteristic $3-\mathrm{Hz}$ generalized spike-and-wave discharge should be absent in the wake EEG, with hyperventilation and photic stimulation, in sleep, and after AEDs are discontinued. Long-term follow-up with both clinical and EEG evidence of continued drug-free remission would be of interest.

Pretreatment EEG and CAE outcome. A study of the relationship between EEG characteristics prior to treatment of $\mathrm{CAE}$, measures of attention, and the response to initial AED treatment with ESM, VPA, or LTG found a predictive relationship between the shortest seizure and response to treatment; the longer the pre-treatment seizure, the more treatment-responsive and better the outcome [1].

Adverse effects of Ethosuximide. While ethosuximide is considered the drug of choice for the control of CAE, its adverse effects may sometimes be serious and no less troublesome than those of valproic acid or lamotrigine. Gastric disturbances are the most common side effects. Next most common are CNS symptoms including fatigue, headache, dizziness, hiccups, and euphoria. Non-dose-related side effects include skin rash, erythema multiforme, systemic lupus erythematosus, eosinophilia, leukopenia, and rarely, pancytopenia. Behavioral changes and psychotic episodes are reported [2].

In a retrospective review of records of $128 \mathrm{CAE}$ patients, the seizure-free rate after ESM (84\%) is significantly higher than that of VPA (62\%) and LTG (53\%) at 3 months but at 9 months, there is no significant difference in seizure-free rates. Rates of normalization of EEG at 12 months and of adverse events are similar for ESM, VPA, and LTG [3]. In a larger controlled clinical trial of the AEDs in 446 children with CAE, almost two thirds of 125 subjects with treatment failure due to lack of seizure control were in the LTG cohort. The VPA cohort had a higher rate of adverse events not seen in the ESM cohort. ESM is considered the optimal initial monotherapy for CAE [4].

\section{References.}

1. Bernson-Leung, et al. Neurology. 2014 May 6;82(18):e158-60.

2. Buchanan RA. Ethosuximide toxicity. In: Woodbury DM, et al., editors. Antiepileptic Drugs. New York: Raven Press; 1972. p. 449-54.

3. Hwang H, et al. Brain Dev. 2012 May;34(5):344-8.

4. Glauser TA, et al. Epilepsia. 2013 Jan;54(1):141-55. 